\title{
On the Synergy Between Adaptive Physical Layer and Multiple-Access Control for Integrated Voice and Data Services in a Cellular Wireless Network
}

\author{
Vincent K. N. Lau, Senior Member, IEEE, and Yu-Kwong Kwok, Member, IEEE
}

\begin{abstract}
In this paper, we propose a novel design to exploit the synergy between the multiple-access control (MAC) layer and the physical layer of a cellular wireless system with integrated voice and data services. As in a traditional design, the physical layer (channel encoder and modulator) is responsible for providing error protection for transmitting the packets over the hostile radio channel, while the MAC layer is responsible for allocating the precious bandwidth to the contending users for voice or data connections. However, a distinctive feature of our proposed design is that in the physical layer, a variable-rate adaptive channel encoder is employed to dynamically adjust the amount of forward error correction according to the time-varying wireless channel state such that the MAC layer, which is a reservation-based time-division multiple-access protocol, is able to make informed decisions as to bandwidth allocation. Specifically, based on the channel state information provided by the physical layer, the MAC protocol gives higher priority to users with better channel states. This novel synergistic mechanism between the two protocol layers can utilize the system bandwidth more effectively.

The multiple-access performance of the proposed scheme is compared with two baseline systems. The first baseline system consists of the same reservation-based MAC protocol but with a traditional fixed-rate physical layer. The second system consists of the same reservation-based $\mathrm{MAC}$ protocol and the same channel adaptive physical layer, but without interaction between the two layers. All three protocols have a request queue, which stores the previous requests that survive the contention but are not allocated information slots. Our extensive simulation results demonstrate that significant performance gains are achieved through the exploitation of the synergy between the two protocol layers.
\end{abstract}

Index Terms-Channel-adaptive, multiple access control, packet scheduling, synergy, wireless media.

\section{INTRODUCTION}

$\mathbf{T}$ RADITIONALLY, based on the layering and information hiding protocol design paradigm, protocol layers are designed separately in order to achieve modularity and portability. Indeed, integrating the functions of protocol layers has recently been widely considered as an attractive approach to improve protocol performance for both wired and wireless networks [1], [26]. In our previous study, we demonstrated the synergistic effect of integrating the physical layer (with variable-rate adaptive

Manuscript received January 4, 2000; revised April 12, 2001. This work was supported by HKU CRCG under Contract 10202523, by HKU URC under Contracts 10203010 and 10203413, and by the Hong Kong Research Grants Council under Contract HKU7024/00E.

The authors are with the Department of Electrical and Engineering, The University of Hong Kong, Hong Kong, China (e-mail: knlau@eee.hku.hk; ykwok@ eee.hku.hk).

Digital Object Identifier 10.1109/TVT.2002.804848 channel encoder and modulator) and cell scheduling for a wireless asynchronous transfer mode network [20]. In this paper, we focus on exploiting the synergy between the multiple-access control (MAC) layer and the physical layer for the reverse link in a cellular wireless network with a star topology providing voice and data services, as shown in Fig. 1.

The classical role of a MAC layer protocol is to judiciously arbitrate the transmission requests of multiple uncoordinated users and assign transmission bandwidth to the users. In a wireless communication system, the MAC layer is particularly important due to the sharing of the precious bandwidth by a dynamically changing population of users with various traffic demands. Thus, the capacity of the network and quality of service depend critically on the performance of the MAC protocol in terms of packet dropping rate, delay, throughput, and utilization. In this regard, a plethora of MAC protocols [5], [10], [13], [22] have been suggested in the literature for supporting integrated voice and data traffics (see [2] for a survey). In general, these previous protocols attempt to accommodate more data users, which do not impose constraints on data delay, by exploiting the silence gaps of the voice users, which require delay-bounded packet transmission and hence enjoy a higher transmission priority than data users in that reservation is allowed for the former but not the latter. However, while sophisticated slot assignment strategies with articulated frame structures are proposed in these methods, none of them considers exploiting the synergy between the MAC protocol and the underlying physical layer. Indeed, these previous protocols are designed and analyzed based on the assumption that packet transmission through the physical channel is error-free. This is a valid assumption in a fixed-line transmission environment, where the performance of the physical layer is essentially error-free and time-invariant. However, in a wireless environment, the error rate of the physical layer is high and the physical layer performance is time-varying. Therefore, the MAC protocol should be matched to the physical layer in order to achieve a better resource utilization. As will be shown in this paper, the knowledge of the channel condition reported to the MAC layer by the physical layer is a very useful component in achieving even higher performance in a wireless communication system where burst errors are the norm rather than exception.

A traditional physical layer delivers a constant throughput in that the amount of error protection incorporated into a packet is fixed without regard to the time-varying channel condition. 


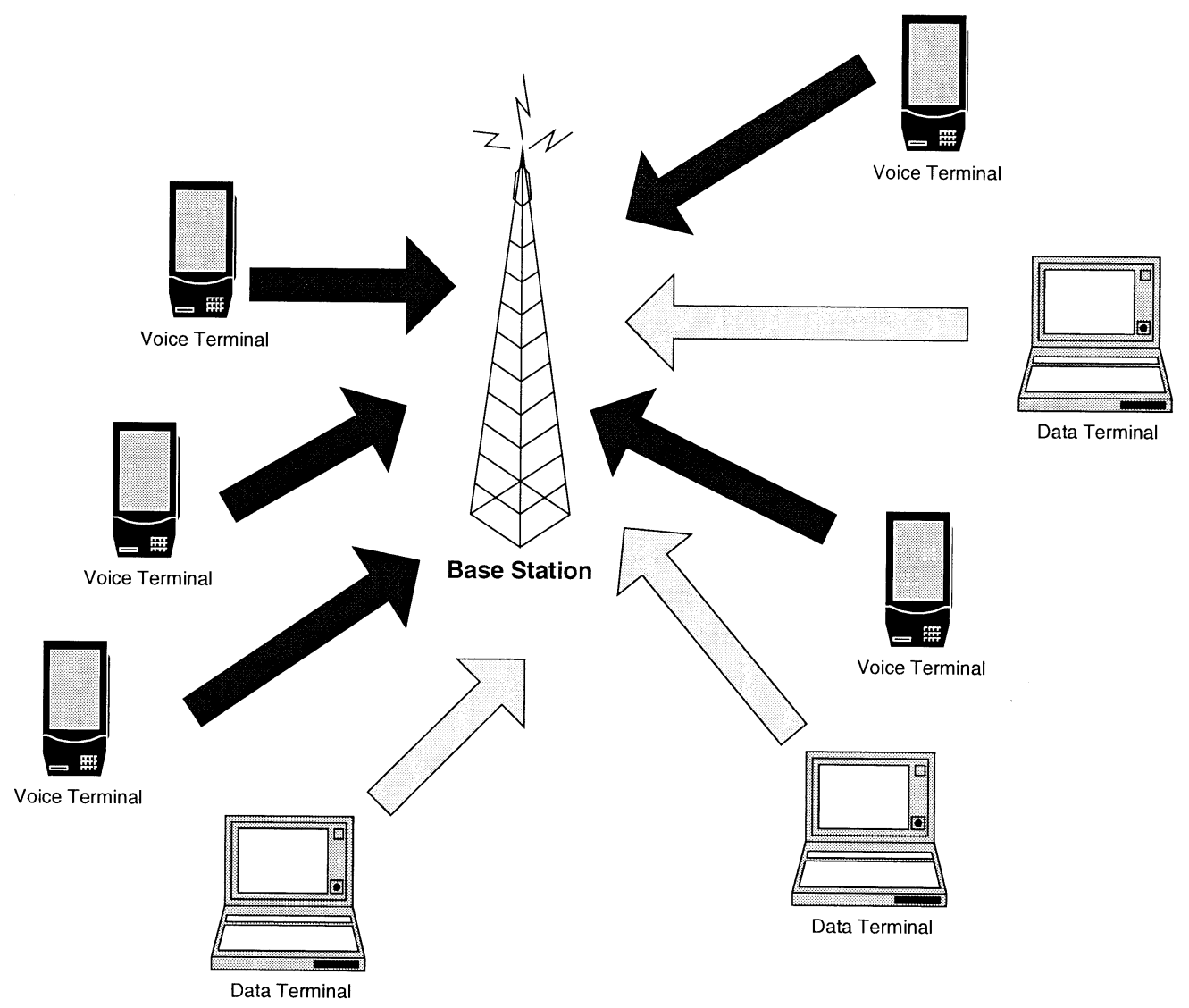

Fig. 1. System model for the reverse-link access of a wireless system with voice and data terminals.

However, to achieve synergy, in our design a variable-rate channel adaptive encoder and modulator are used in the physical layer [3], [19], [21]. Specifically, a low-capacity feedback channel is employed to convey estimated channel state information (CSI) to the transmitter. Thus, under good channel condition (i.e., signal attenuation is low), the amount of protection added in the transmitter is reduced to boost the throughput. On the other hand, more protection is added when the channel condition becomes worse. Using this dynamically adjusted level of protection, the bit error rate (BER) is maintained at a targeted level over a range of channel conditions. ${ }^{1}$ It has been shown [21] that a significant gain in the average rate can be achieved in these adaptive channel coding schemes. Our major contribution in this paper is to propose a jointly adaptive MAC protocol, called CHARISMA (channel adaptive reservation-based isochronous multiple access), to illustrate the potential benefit of combined design of the physical layer and the MAC layer. As will be detailed in Section III-C, both the physical layer and the MAC layer of CHARISMA are jointly adaptive to the CSI. To demonstrate the performance gain derived from the joint design, we compare the performance of our proposed scheme with two baseline systems, as shown in Figs. 2 and 3. Baseline System-I is also a Dynamic-time-division multiple-access (TDMA)-based MAC layer, but a constant throughput physical layer is used. In Baseline System-II, a

\footnotetext{
${ }^{1}$ When channel adaptive modulation and coding are employed, the penalty during poor channel conditions is therefore a lower offered throughput instead of a higher error rate.
}

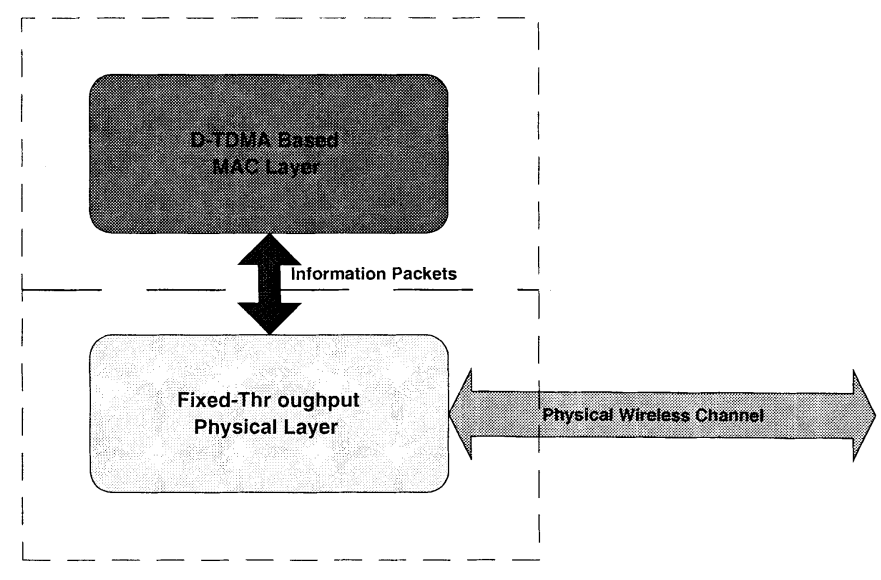

Base Station (Baseline System-I)

Fig. 2. Baseline System-I: fixed-rate channel encoder.

variable-rate adaptive physical layer is incorporated with a D-TDMA-based MAC layer. In both baseline systems, there is no interaction between the physical layer and the MAC layer, unlike that in the CHARISMA protocol (see Fig. 4). Our extensive simulation results indicate that for the same quality of service level, CHARISMA achieves a considerably greater capacity.

Deriving synergy between protocol layers is a relatively unexplored research topic. In [14], an adaptive modulation reservation TDMA scheme is proposed. However, such a scheme is 


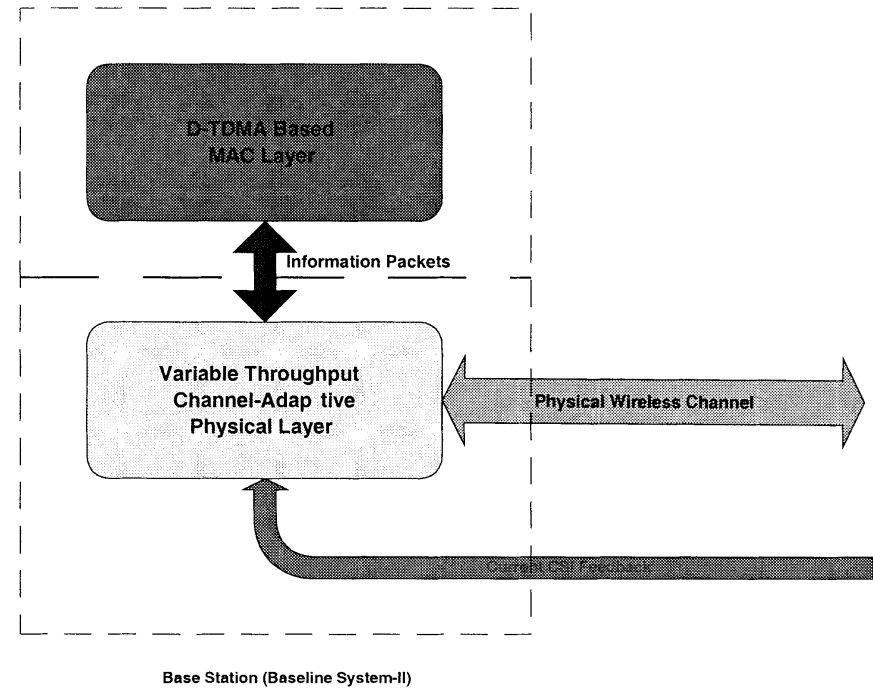

Fig. 3. Baseline System-II: adaptive channel encoder.

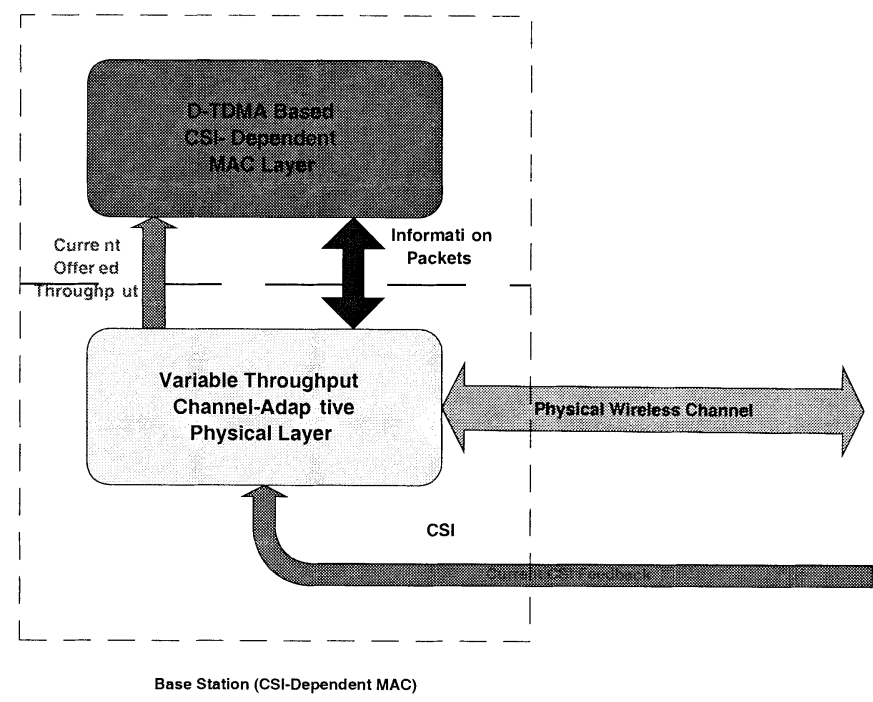

Fig. 4. CHARISMA: adaptive channel encoder with MAC interaction.

essentially a straightforward cascade of the MAC layer with an adaptive modulator; thus, no synergy is exploited. ${ }^{2}$ In [6], a channel state dependent (CSD) packet scheduling scheme is suggested to improve the uplink performance of multiplexing several transmission control protocol connections in a wireless network. Their scheme is therefore different from our proposed protocol in two important aspects: 1) their scheme is targeted for transport layer, while our scheme is designed for MAC layer; and 2) their scheme is to be used for the downlink, while our scheme is for the uplink. Furthermore, their channel state estimation, which is solely based on observing the delays of ACK packets, is inevitably less accurate. Finally, a CSD bandwidth assignment algorithm is proposed in [12]. However, only simple assignment schemes (monopolistic allocation and equal sharing) are considered therein.

This paper is organized as follows. In Section II, we describe the channel model and the physical layer design. In Section III,

${ }^{2}$ The scheme in [14] is similar to our Baseline System-II. we describe the proposed TDMA-based MAC protocol with interaction with the physical layer. Experimental results, in terms of packet loss probability (for voice users), average delay, and throughput (for data users), are described in Section IV. The last section concludes this paper.

\section{VARIABLe-Rate ChanNEL-Adaptive Physical LAYER}

\section{A. Wireless Channel Model}

The wireless communication environment considered in this paper is the uplink situation of a wireless system where a number of mobile terminals contend to transmit information to a base station, as illustrated in Fig. 1. The wireless link between a mobile terminal and the base station is characterized by two components: the fast fading component and the long-term shadowing component. Fast fading is caused by the superposition of multipath components and is therefore fluctuating in a very fast manner (on the order of a few milliseconds). Long-term shadowing is caused by terrain configuration or obstacles. Let $c(t)=c_{l}(t) c_{s}(t)$ be the combined channel fading where $c_{l}(t)$ and $c_{s}(t)$ are the long-term and short-term fading components, respectively. Without loss of generality, we assume $\mathcal{E}\left[c_{s}^{2}(t)\right]=1$, where $\mathcal{E}[]$ denotes the expected value of a random variable. The probability distribution of $c_{s}(t)$ follows the Rayleigh distribution. In this paper, we assume that the maximum mobile speed is $80 \mathrm{~km} / \mathrm{h}$, and hence the Doppler spread [24] $f_{d} \approx 100 \mathrm{~Hz}$ at a carrier frequency of $2 \mathrm{GHz}$. It follows that the coherent time $T_{c}$ is approximately given by

$$
T_{c} \approx \frac{1}{f_{d}}
$$

which is about $10 \mathrm{~ms}$.

The long-term fading component $c_{l}(t)$ is also referred to as the local mean [24], which, as shown by field test measurement [18], obeys the log-normal distribution. Since mobile terminals are scattered geographically across the cell and are moving independently of each other, we assume that the channel fading experienced by each mobile terminal is independent of each other.

\section{B. Frame Structures}

Frequency-division duplexing (FDD) is used in this paper. All the uplink and downlink frames are synchronized in the same way as those traditional MAC schemes. Specifically, the base station and all the mobile stations have a common and synchronized timing reference. Fig. 5 shows the frame structure for the uplink and the downlink in the considered wireless communication system, which is TDMA-based. An uplink frame is used for transmitting information from the mobile terminals to the base station; as such, the multiple-access problem has to be tackled. By contrast, a downlink frame is used for transmitting information from the base station to the mobile terminals and is, therefore, a broadcasting channel such that no contention is involved. Our focus in this paper is on the multiple access aspect of the uplink.

In the uplink, a frame is divided into three subframes, as illustrated in Fig. 5: the request subframe, traffic subframe, and pilot symbol subframe. Specifically, there are $N_{r}$ minislots in the request subframe for voice request reservation and data request contention. Note that a data user is not allowed to make 


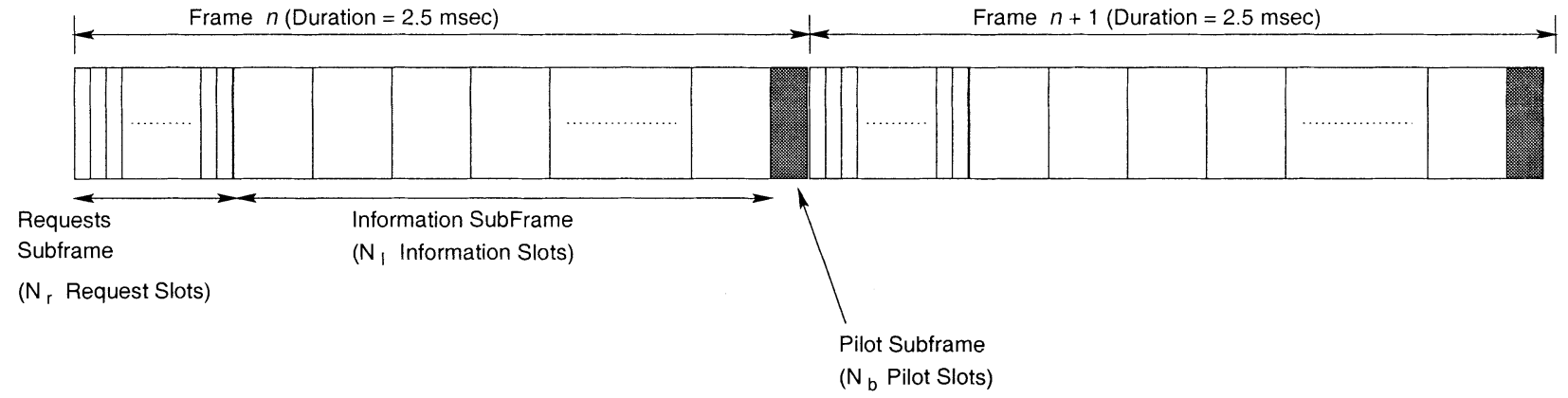

(a) uplink frame structure

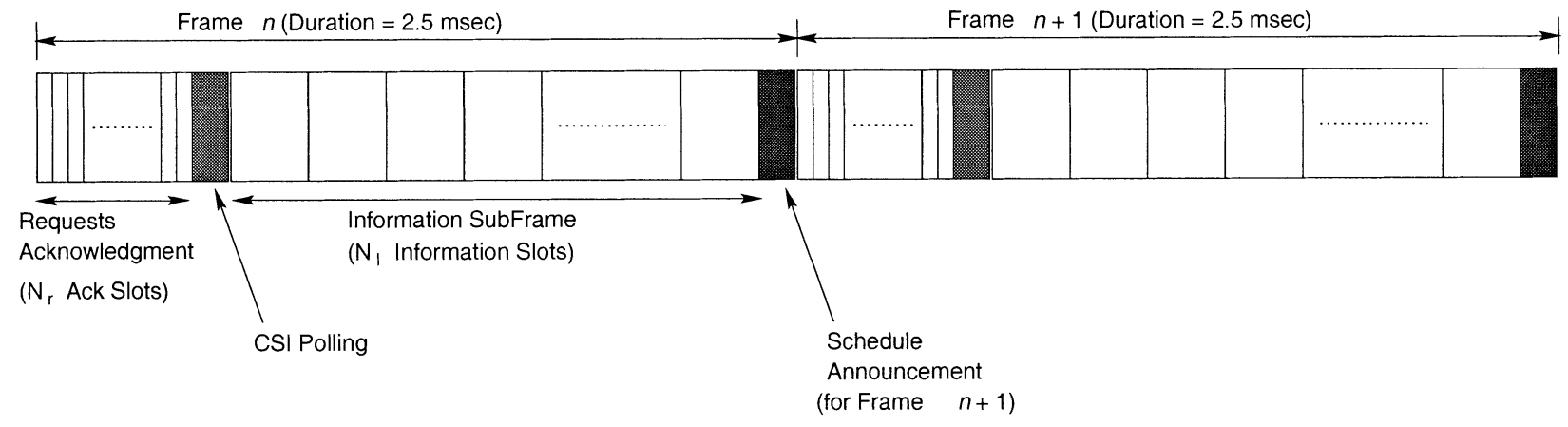

(b) downlink frame structure

Fig. 5. Frame structures of the proposed CSI-dependent MAC protocol.

a reservation in the sense that even if a data user successfullly seizes an opportunity to transmit in the current frame, he has to contend again in the next frame if he has more data to send. There are $N_{i}$ information slots in the traffic subframe for the transmission of voice or data packets. Finally, there are $N_{b}$ slots in the pilot symbol subframe, the function of which will be elaborated in detail in Section III-C. The frame duration is $2.5 \mathrm{~ms}$. Such a short frame duration has the advantage of shorter delay and is practicable in wide-band systems [23].

On the other hand, a downlink frame is similarly partitioned into four subframes: the acknowledgment subframe, poll-for-CSI subframe, traffic subframe, and announcement subframe. The frame duration is also $2.5 \mathrm{~ms}$, and the number of slots in the subframes are given by $N_{r}, N_{b}, N_{i}$, and $N_{i}$, respectively. The functionality and operation of each subframe are described in Section III.

\section{Variable-Rate Physical Layer}

Redundancy is incorporated into the information packet for error protection. To exploit the time-varying nature of the wireless channel, a variable rate channel-adaptive physical layer is employed, as illustrated in Fig. 6(a). CSI $c(t)$, which is estimated at the receiver, is fed back to the transmitter via a low-capacity feedback channel. Based on the CSI, the level of redundancy and the modulation constellation applied to the information packets are adjusted accordingly by choosing a suitable transmission mode. Thus, the instantaneous rate is varied according to the instantaneous channel state. A number of schemes have been proposed for high bandwidth efficiency situations [15]-[17]. It is shown by simulation and analysis
[19] that an adaptive bit-interleaved trellis-coded modulation scheme (ABICM) achieves significant performance gain compared to other schemes. Furthermore, ABICM has the advantage of flexible design. Therefore, we adopt ABICM in CHARISMA as an illustration. For details of the design and analysis of ABICM, please refer to [19].

In our study, a six-mode ABICM, which is a design tradeoff between performance and complexity, is employed [19]. Transmission modes with normalized throughput ${ }^{3}$ varying from $1 / 2$ to five are available depending on the channel condition.

We assume that the coherent time of the short-term fading is around $10 \mathrm{~ms}$, which is much longer than an information slot duration. Thus, the CSI remains approximately constant within a frame, and it follows that the transmission mode for the whole frame is determined only by the current CSI level. Specifically, transmission mode $q$ is chosen if the feedback CSI $\hat{c}$ falls within the adaptation thresholds $\left(\zeta_{q-1}, \zeta_{q}\right)$. Here, the operation and the performance of the ABICM scheme is determined by the set of adaptation thresholds $\left\{\zeta_{0}, \zeta_{1}, \ldots\right\}$. In this paper, we assume that the ABICM scheme is operated in the constant BER mode [19]. That is, the adaptation thresholds are set optimally to maintain a target transmission error level over a range of CSI values. When the channel condition is good, a higher mode could be used and the system enjoys a higher throughput. On the other hand, when the channel condition is bad, a lower mode is used to maintain the target error level at the expense of a lower transmission throughput. This is because we would like to exploit the instantaneous variation of the offered throughput in the physical layer. Usually, the quality of service $(\mathrm{Q} O S)$ requirement will

\footnotetext{
${ }^{3}$ Normalized throughput refers to the number of information bits carried per modulation symbol.
} 


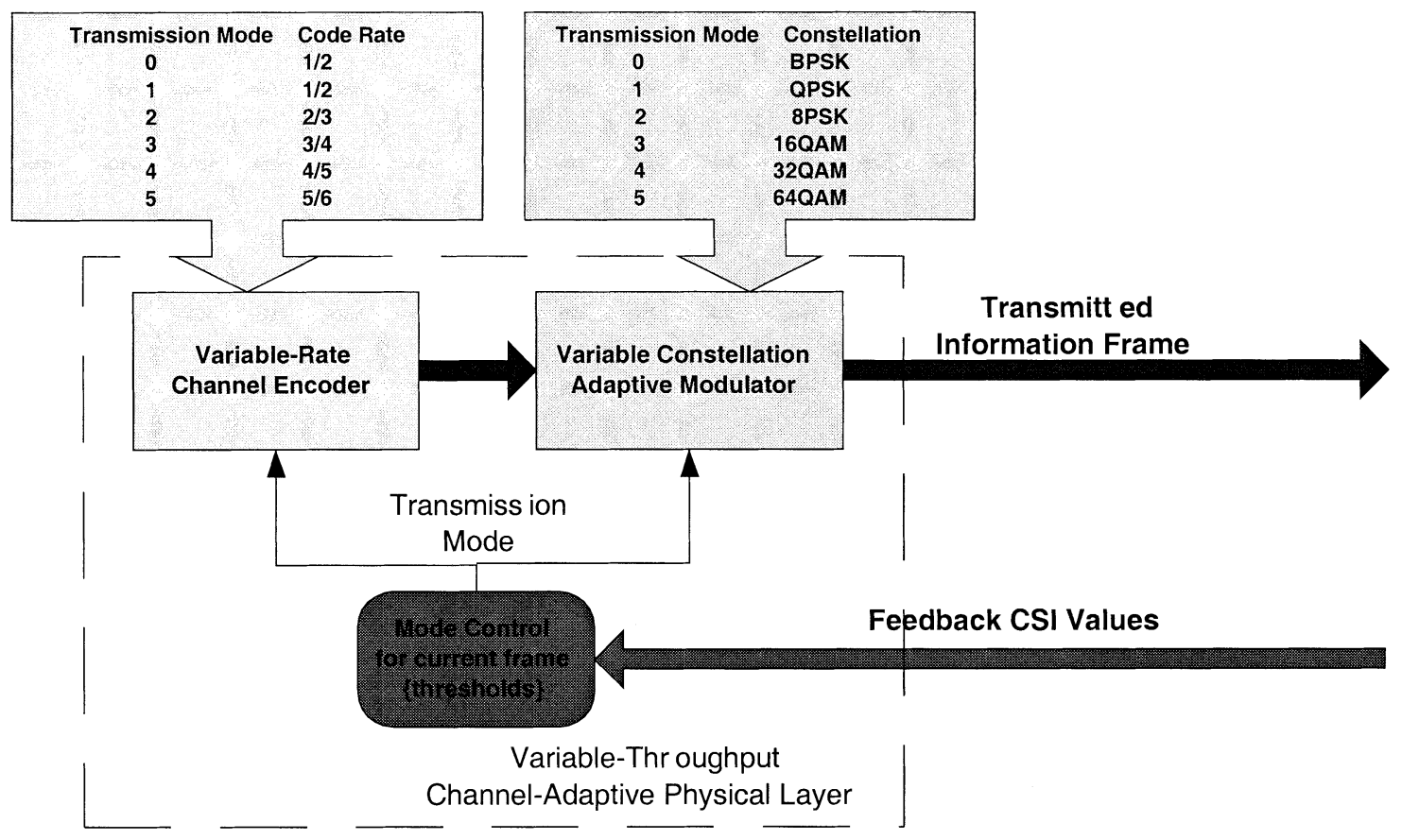

Fig. 6. A conceptual block diagram of the variable-rate channel adaptive physical layer.

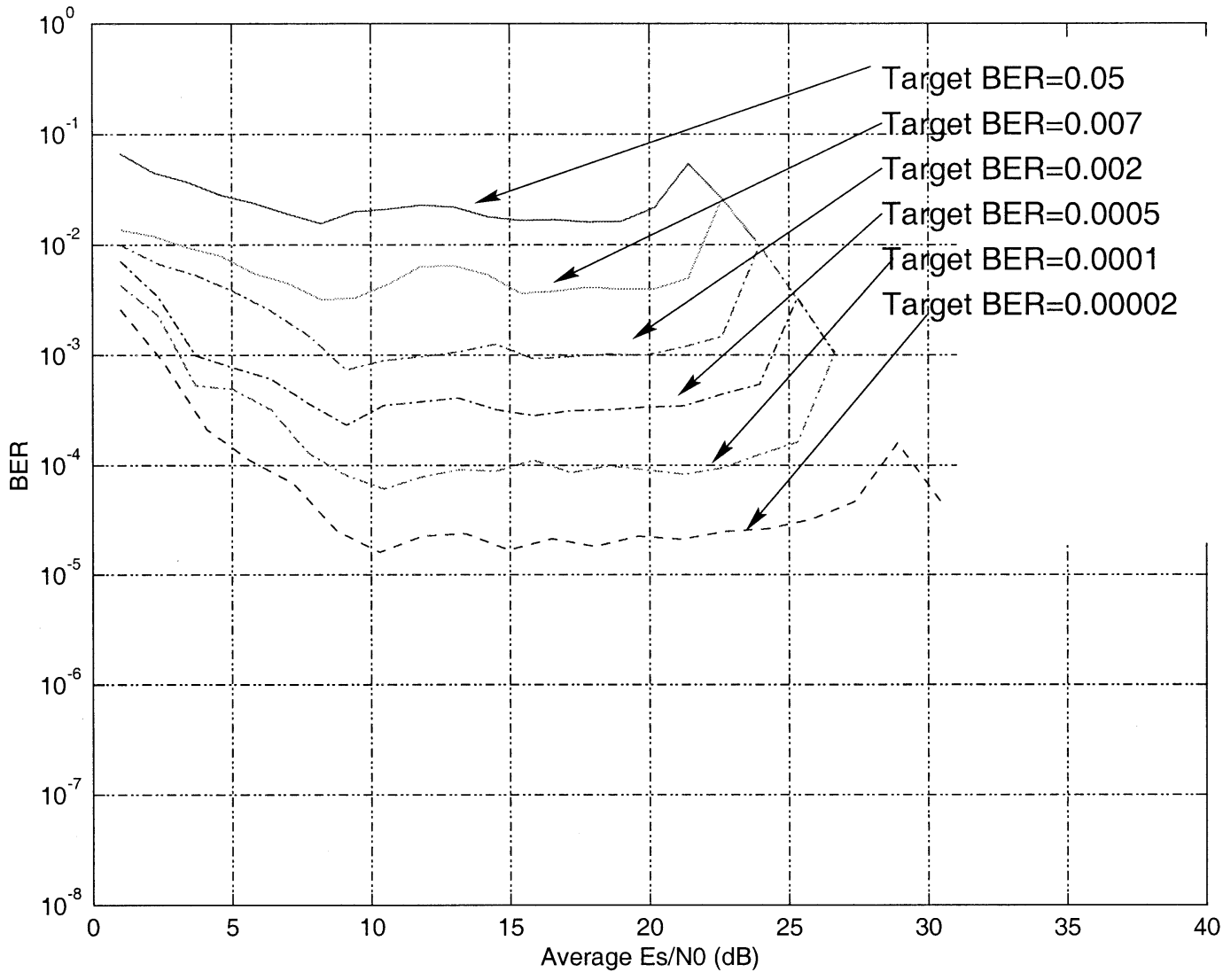

Fig. 7. ABICM: instantaneous BER and the adaptation range.

specify a target error level. The additional gain in "QoS utilization" during good channel states by having a lower than target BER level is not large. However, with constant BER control, the additional gain in "QoS utilization" could be much higher because the "lower than target BER" is now turned into a temporal boost in throughput. The MAC layer synergy is actually derived from the instantaneous fluctuations of the offered throughput. This is illustrated in Fig. 7.

Given the above considerations about the channel state, the instantaneous throughput offered to the MAC layer $\rho$ is also 


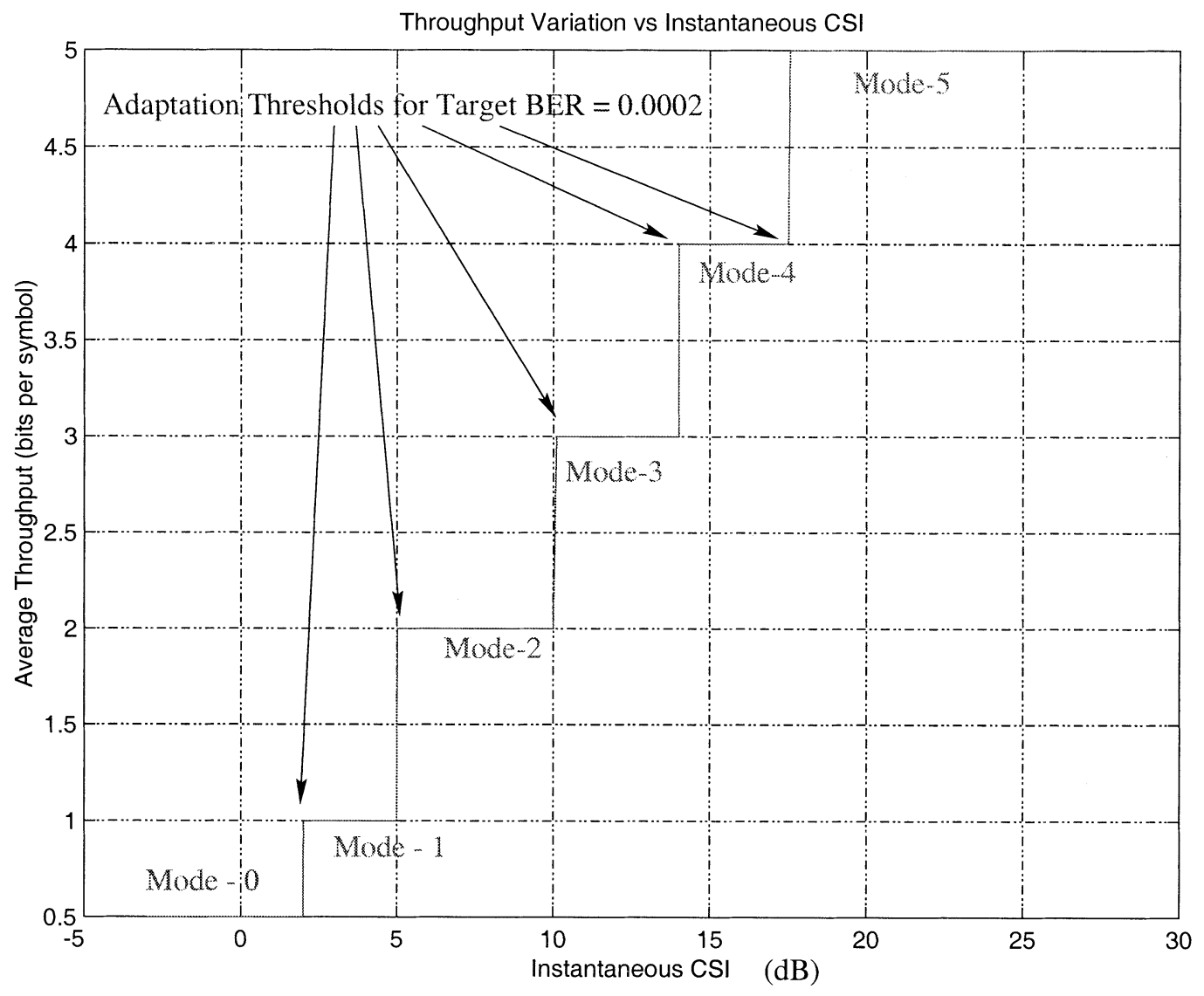

Fig. 8. ABICM: instantaneous throughput versus CSI.

variable and is therefore a function of the CSI $c(t)$ and the target BER $P_{b}$, denoted by $\rho=f_{\rho}\left(c(t), P_{b}\right)$. Fig. 8 illustrates the variation of $\rho$ with respect to the CSI.

\section{THE CHANNEL-ADAPTIVE MAC LAYER}

In the following, we illustrate the potential benefit of exploiting interprotocol layer synergy through our proposed D-TDMA-based MAC protocol as an example. The wireless communication system consists of only voice and data terminals. As in most previous studies, it is assumed that voice packets are delay sensitive while data packets are delay insensitive.

\section{A. A Brief Survey of TDMA-Based MAC Protocol}

D-TDMA was first introduced for satellite communications [8], [9] and has been proposed recently as a candidate MAC protocol for the third-generation wireless communication systems [5], [22]. There are many variations of D-TDMA-based MAC protocols, such as RAMA [5], RMAV [13], and DRMA [22]. Time on the channel is divided into a contiguous sequence of TDMA frames, which are subdivided into request slots and information slots. The information slots are sometimes further classified into voice and data slots. There are two types of packets being transmitted in the channel: the request packet and the information packet. A request packet is used for the request of an information slot (either voice or data slots). It often includes only a very small amount of information-namely, the origin and the destination addresses - and is therefore usually much shorter than an information packet. The request subframe is usually operated using the slotted-ALOHA protocol.

A terminal entering a new voice talkspurt or generating a new stream of data packets transmits an appropriate request packet in one of the request slots of the next frame. If there is more than one packet transmitted in the same request slot, collision occurs. Depending upon whether the capture effect [4], [25] is considered, one or none of the requests will be correctly received (we considere cases both with and without capture). At the end of each request slot, the successful or unsuccessful request will be identified and broadcast by the base station. An unsuccessful user can retry in the next request slot. On the other hand, a successful user then transmits his information packet in the corresponding information slot in the current frame.

In all previous schemes, bandwidth allocation in the MAC layer for a certain traffic type is essentially based only on a first-come, first-served strategy. Most importantly, these protocols do not take into account the CSI in the bandwidth allocation process. This motivates our proposed new MAC protocol, CHARISMA, which works by adaptively assigning information slots to users based on their CSI ranking. Before we discuss the proposed CHARISMA algorithm, we first introduce the source model for the voice and data users.

\section{B. Source Models}

The wireless system considered in this paper is aimed to support integrated voice and data services. As such, we assume that 


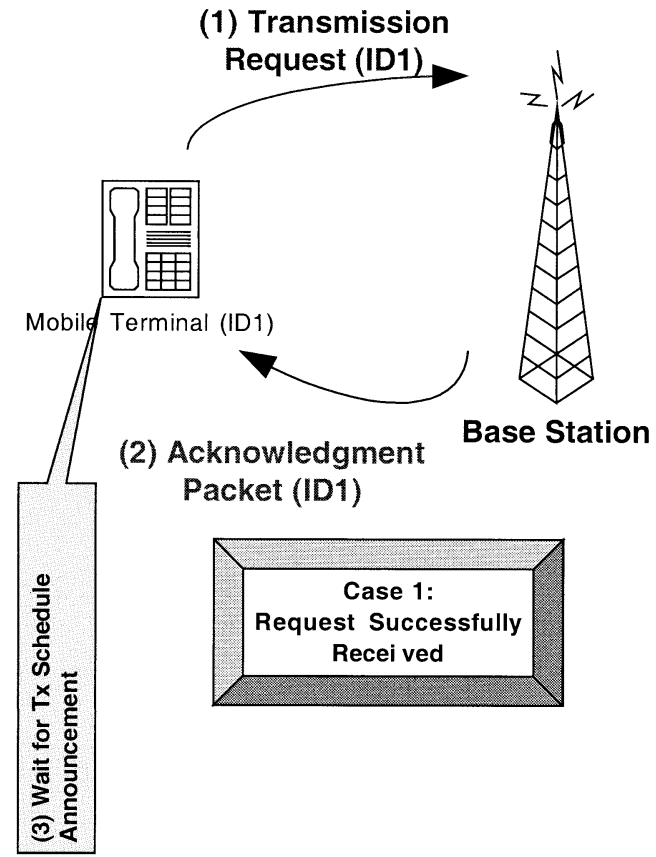

Fig. 9. CHARISMA: operation mechanisms of request and acknowledgment.

there are only two types of mobile terminals: the voice terminal and the data terminal. Voice packets are assumed to be delay sensitive, while data packets are assumed to be delay insensitive. Thus, voice packets are labeled with deadlines. A voice packet will be dropped by a voice terminal if the deadline expires before being transmitted. Such packet dropping has to be controlled to within a certain limit (e.g., below $1 \%$, as indicated in [11]) in order that the QoS to the voice users is still acceptable. The source and contention models are summarized below.

- Voice Source Model: The voice source is assumed to be continuously toggling between the talkspurt and silence states. The duration of a talkspurt and a silence period are assumed to be exponentially distributed with means $t_{t}$ and $t_{s} \mathrm{~s}$, respectively (as indicated by the empirical study in [22], $t_{t}=1$ and $t_{s}=1.35$ ). We assume that a talkspurt and a silence period start only at a frame boundary.

- Data Source Model: The arrival time of data generated by a data terminal is assumed to be exponentially distributed with mean equal to $1 \mathrm{~s}$. The data size, in terms of number of packets, is also assumed to be exponentially distributed with mean equal to $m_{d}$ packets. This is a simplifying assumption adopted in most previous literatures [4], [5], [25] for easy analysis. Again we assume that the packets arrive at a frame boundary.

- Terminal Contention Model: As in most previous studies, to avoid excessive collisions, even if a voice or data user has some packets waiting to be sent, the user will attempt to send a request at a request minislot only with a certain permission probability. The permission probability for voice and data users is denoted by $p_{v}$ and $p_{d}$, respectively. 4

\footnotetext{
${ }^{4}$ The values 0.3 and 0.2 are chosen for $p_{v}$ and $p_{d}$, respectively, based on the results of [9] and [10]. The idea is to give priority to voice users by granting them a high permission probability to retransmit the request packets after contention.
}

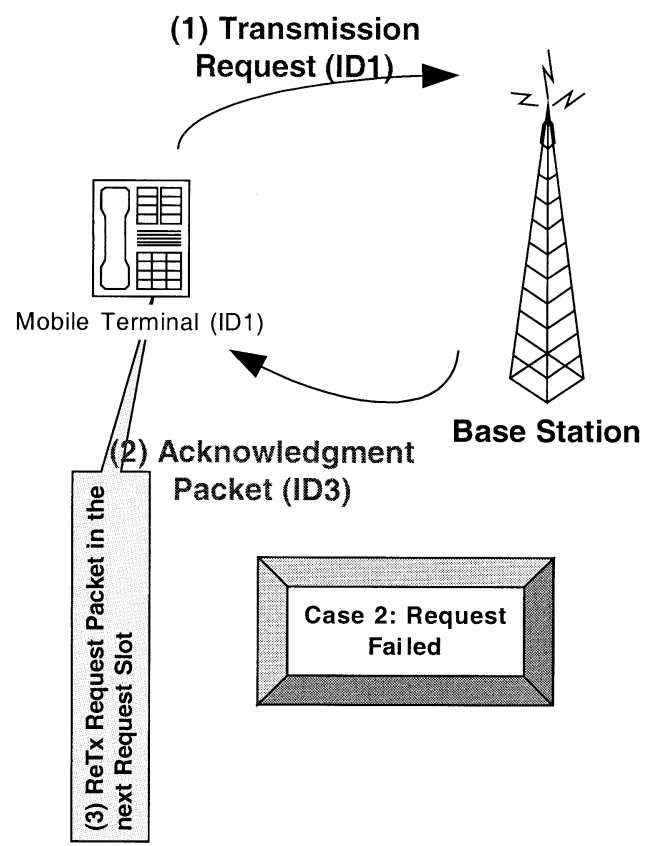

\section{Operations of CHARISMA}

In this section, we describe the design of CHARISMA, a novel channel state dependent MAC protocol that works closely with the variable-throughput channel-adaptive physical layer (described in Section II). The operation of the CHARISMA protocol is divided into two phases: the request phase and the transmission phase. In the request phase, mobile terminals that have voice or data packets to transmit will send a request packet in one of the request slots, governed by the respective permission probability. The request packet is very short, occupying only a minislot, as illustrated in Fig. 11(a). It contains the mobile terminal ID, request type (voice or data), packet deadline, number of information packets desired to transmit as well as some pilot symbols. If more than one mobile terminals send request packets in the same request slot, collision occurs, and all the request packets are lost if capture effect is not considered (if capture is considered, the request with the highest signal energy may be successfully received). After each request slot, an acknowledgment packet will be broadcast from the base station through the acknowledgment slot in the downlink frame, as illustrated in Fig. 9. The acknowledgment packet contains only the successful request packet ID. Mobile terminals that fail to receive an acknowledgment will retransmit the request packet in the next request slot, again governed by the permission probability. On the other hand, successfully acknowledged users will wait for an announcement on the allocation schedule from the base station.

Unlike traditional MAC protocols, the base station will collect all requests in the current request phase as well as the backlog requests from the previous frames. All the requests will be assigned priorities that are computed according to the deadline, CSI, and service type (voice or data), as well as the waiting time of the request (i.e., the number of elapsed frames since the request is acknowledged). The timeslot allocation algorithm is conceptually depicted in Fig. 10. Since the physical 


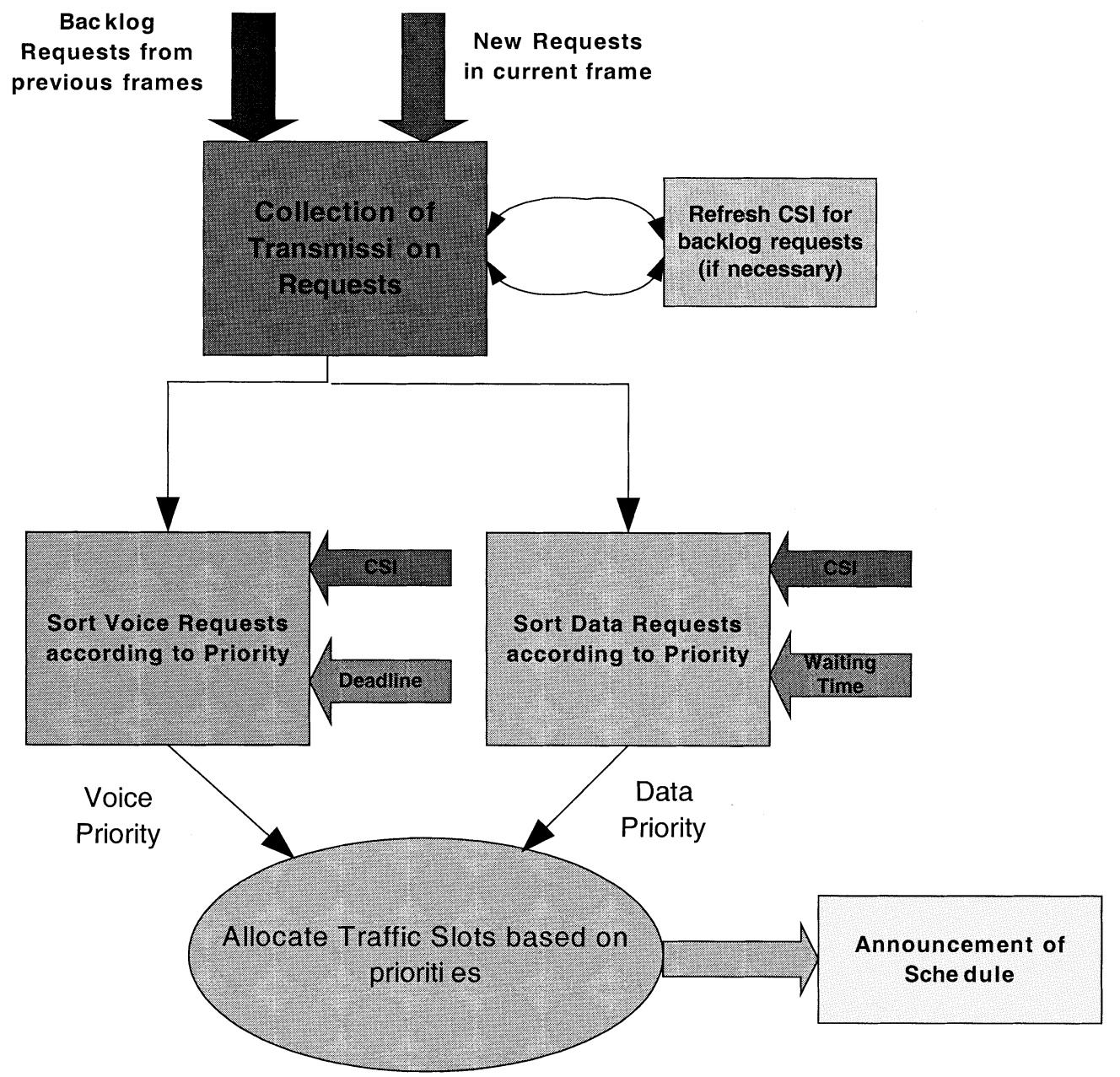

Fig. 10. CHARISMA: the time-slot allocation mechanism.

layer offers a variable rate that is dependent on the CSI, the rationale behind the CHARISMA MAC protocol is to give higher priority to the mobile terminals that are in better channel condition in the bandwidth allocation process. The motivation of this strategy is that a user with better channel condition, with the support of the variable-rate channel encoder, can enjoy a larger transmission rate and therefore can use the system bandwidth more effectively. Nevertheless, for the sake of fairness, information slots should also be allocated to mobile terminals that are approaching their deadlines, despite their possibly worse channel states; otherwise, the queued information packets will be dropped. Specifically, the priority metric of the $i$ th request (which may be a new request or a backlog request) $\mu_{i}$ is given by (2), shown at the bottom of the page, where $T_{d}^{(i)}, T_{w}^{(i)}, \lambda_{v}, \lambda_{d}, \beta_{v}, \beta_{d}$, and $\Delta V$ are the deadline, waiting time the forgetting factors of the voice and data requests, and priority offset assigned to the voice users, respectively. As can be seen from (2), a higher priority will be assigned to requests with a higher throughput (indicated in the first term) as well as requests with a urgent deadline or long waiting time (indicated in the second term). Thus, in the allocation phase, information timeslots are allocated to all service requests according to the priority metrics. If there are not sufficient timeslots to service all requests, remaining requests are queued and reconsidered in the next frame. ${ }^{5}$ After the request phase, the results of information timeslot allocation will be broadcast in the announcement subframe of the downlink frame. The announcement packet contains the timeslot allocation schedule as well as the transmission mode, as illustrated in Fig. 11(b). Mobile terminals will then start to transmit information packets on the allocated time slots.

If the deadline for a remaining request has expired, this request will not be queued anymore. The information packet at the mobile terminal will be dropped.

$$
\mu_{i}= \begin{cases}g_{v}\left(C S I^{(i)}, T_{d}^{(i)}\right)=f_{\rho}\left(C S I^{(i)}, P_{b}\right)+\lambda_{v}\left(T_{d}^{(i)}\right)^{-\beta_{v}}+\Delta V & \text { for voice request } \\ g_{d}\left(C S I^{(i)}, T_{w}^{(i)}\right)=f_{\rho}\left(C S I^{(i)}, P_{b}\right)+\lambda_{d}\left(T_{w}^{(i)}\right)^{\beta_{d}} & \text { for data request }\end{cases}
$$




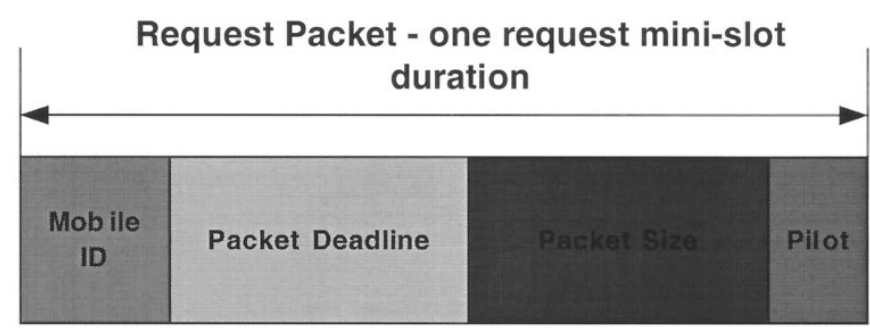

(a) request packet format

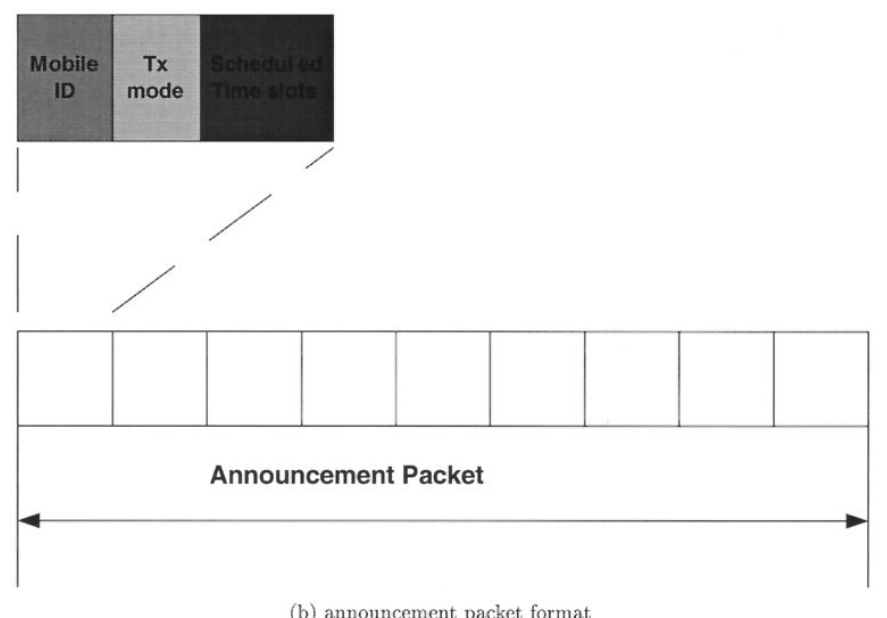

Fig. 11. Formats of the request and announcement packets. The packets illustrate the payload composition only.

The CHARISMA protocol is reservation-based for voice users only. As mentioned earlier, for a data user, even if information time slots have been assigned for its successfully acknowledged request, the allocation is meant only for the current frame, and the user has to initiate another request if there are still some more data packets to be sent. By contrast, for a voice user, when information time slots have been assigned for its successfully acknowledged request, additional requests will be automatically generated by the base station (hence, reservation) periodically at 20-ms time intervals (the voice packet period). Thus, the voice user no longer needs to contend for request slots for the whole remaining time in the current talkspurt. By avoiding unnecessary requests, the advantage of this reservation strategy is to reduce the contention collisions by the request slots. The CHARISMA protocol is outlined below in pseudocode format.

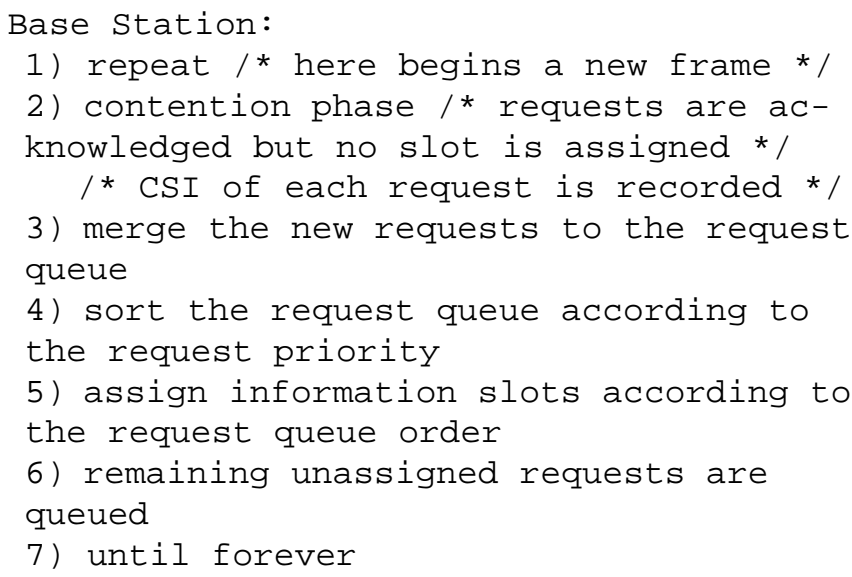

\section{CSI Determination}

A critical component in the proposed CHARISMA MAC protocol is the determination of the current CSI for each user. As mentioned earlier, we assume that the coherent time for short-term fading is around $10 \mathrm{~ms}$, as illustrated in Section II, while the frame duration is only $2.5 \mathrm{~ms}$. Thus, the CSI remains approximately constant for a number of frames. For a new request, known pilot symbols are embedded in the request packets so that the CSI can be estimated at the base station; most importantly, this estimated CSI is valid even for the next frame duration. However, for a backlog request, the estimated CSI value obtained previously during a past request phase may be obsolete; thus, a mechanism is needed to obtain update the CSI. The CSI updating procedure used in the CHARISMA protocol is illustrated in Fig. 12(a).

At the beginning of each frame, the base-station short-lists $N_{b}$ backlog requests (those with their CSI values expired) according to their priorities. A CSI polling packet is then broadcast to the mobile terminals in the CSI polling subframe. The CSI polling packet contains the mobile terminal IDs that are short-listed by the base station. The structure of the polling packet is shown in Fig. 12(b). Mobile terminals listed in the polling packet transmit known pilot symbols at the appropriate pilot symbol slot according to the order specified in the polling packet. Thus, the base station can update the higher priority backlog requests' CSI values, which are valid for two consecutive frames. The estimated CSI value is used to determine the transmission mode in the physical layer as well as to determine the priority of the request in the MAC layer.

\section{PERformance Results}

We have implemented the proposed CHARISMA protocol in a simulator. Two baseline systems are also considered for comparison. System-I is composed of a traditional TDMA-based MAC and fixed-throughput physical layer, while System-II is composed of a traditional MAC protocol and a variable-throughput channel-adaptive physical layer. All three schemes are operating at the same average error level at the same power. That is, the physical layer of System-I offers a fixed throughput to the MAC layer. The physical layers of System-II and the CHARISMA scheme are channel-adaptive and offer the same average throughput to the MAC layer. In both System-I and System-II, the MAC layer and the physical layer are isolated, and there is no interaction between them. In fact, System-II is used as a control to illustrate that the reported gain of CHARISMA is not due to increased average throughput of the adaptive physical layer but due to the interaction between the physical layer and the MAC layer. This would be demonstrated by observing the performance gain of our scheme relative to System-II. The operation of System-I and System-II is briefly outlined below. In our simulation study, we assume a transmission bandwidth of $320 \mathrm{kHz}$ for the TDMA frames. The bit rate of the speech terminals is $8 \mathrm{kbps}$, which conforms to the values in GSM and CDMA systems. Table I summarizes the parameters we used.

System-I: In System-I, the physical layer offers a constant throughput to the MAC layer. The MAC layer assigns time slots to voice terminals on a first-come, first-served basis without regard to the channel condition detected at the physical layer. Specifically, a traditional slots assignment strategy is used in 


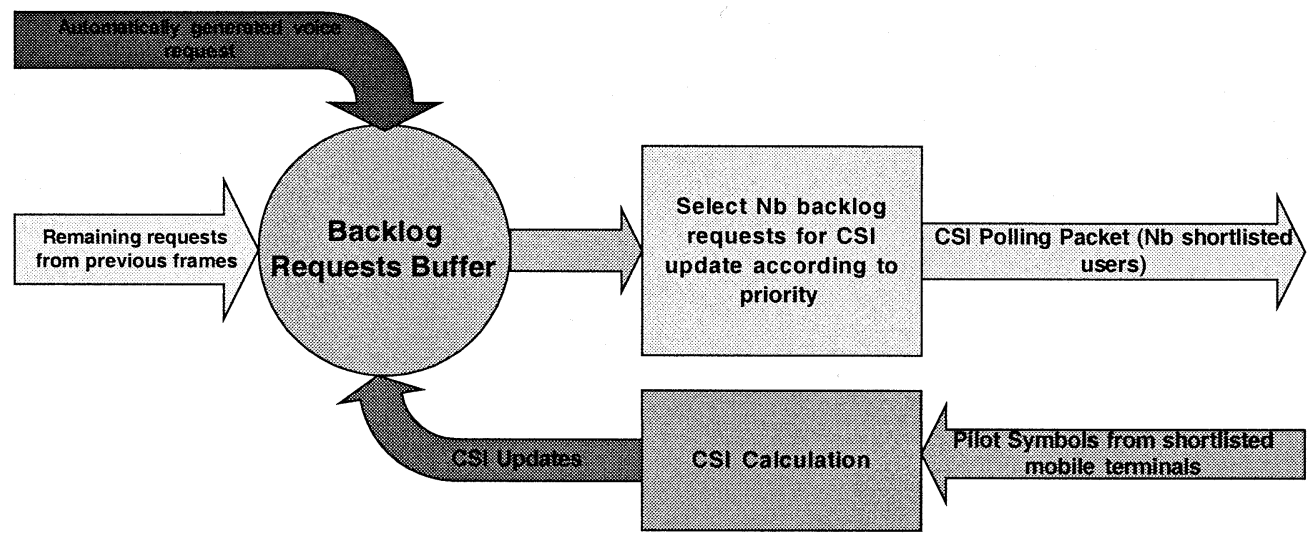

(a) CSI updating mechanism for backlog requests

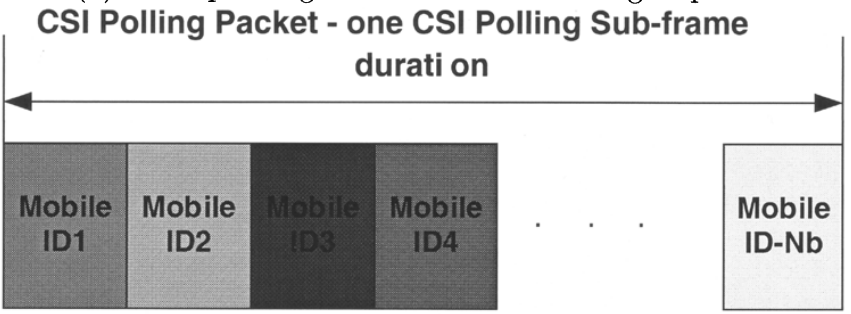

(b) structure of CSI-polling packet

Fig. 12. Illustration of CSI refresh mechanism for backlog requests. The packet illustrates the payload composition only.

TABLE I

SIMULATION PARAMETERS

\begin{tabular}{ll}
\hline Parameter & Value \\
\hline$N_{r}$ & 13 \\
$N_{i}$ & 8 \\
$N_{b}$ & 5 \\
$\lambda_{v}$ & 20 \\
$\lambda_{d}$ & 20 \\
$\beta_{v}$ & 0.5 \\
$\beta_{d}$ & 0.5 \\
$\Delta V$ & 40 \\
$t_{t}$ & $1000 \mathrm{msec}$ \\
$t_{s}$ & $1350 \mathrm{msec}$ \\
$p_{v}$ & 0.3 \\
$p_{d}$ & 0.2 \\
data rate (data users) & $19.2 \mathrm{Kbps}$ \\
number of frames & $2 \times 10^{6}$ \\
\hline
\end{tabular}

that whenever a request is successfully received in the request phase, information slots, if any, are immediately assigned to the requests. After a voice user has successfully reserved an information timeslot in the current frame, the user can use a timeslot in each frame every $20 \mathrm{~ms}$ until the current talkspurt terminates. A data user will be allocated information slots only if there are remaining information slots in the current frame. The data requests are considered also on a first-come, first-served basis.

System-II: System-II employs the same MAC layer as in System-I. However, it differs from System-I in that a variablethroughput channel-adaptive physical layer is used. However, although a variable rate is offered to the MAC layer, there is no interaction between the MAC layer and the physical layer. In other words, the MAC layer is not aware of the current situation in the physical layer in the process of bandwidth allocation. However, with the help of the channel-adaptive physical layer, System-II has twice the average offered throughput compared to System-I.

It is also interesting to investigate the performance of the proposed CHARISMA protocol under situations with and without capture. Thus, the capture process is also implemented. Specifically, suppose there are $k$ requests, with signal power denoted by $P_{1}, P_{2}, \ldots, P_{k}$, contending for a request minislot. A request $j$ can be captured if [4], [25]

$$
\frac{P_{j}}{\sum_{i \neq j} P_{i}}>\gamma
$$

where $\gamma$ is the SNR threshold.

\section{A. Performance for Voice Terminals}

Since the quality of voice communication is determined by the average packet loss probability, we characterize the performance of a voice terminal by packet loss probability $P_{\text {loss. }}$. Note that the packet loss probability is contributed by two factors: packet dropping and packet transmission error. On one hand, a voice packet is delay sensitive, and hence voice packets are labeled with deadlines. A voice packet has to be discarded if its delay exceeds the deadline. ${ }^{6}$ The performance of packet-loss probability versus the number of active voice users $\left(N_{v}\right)$ and active data users $\left(N_{d}\right)$ is shown in Figs. 13 and 14 for CHARISMA, System-I, and System-II.

As can be seen, in all test cases, System-II is superior to System-I in terms of the packet-loss probability because it enjoys

\footnotetext{
6In this paper, the deadline of a voice packet is assumed to be $20 \mathrm{~ms}$ after it is generated by the source.
} 


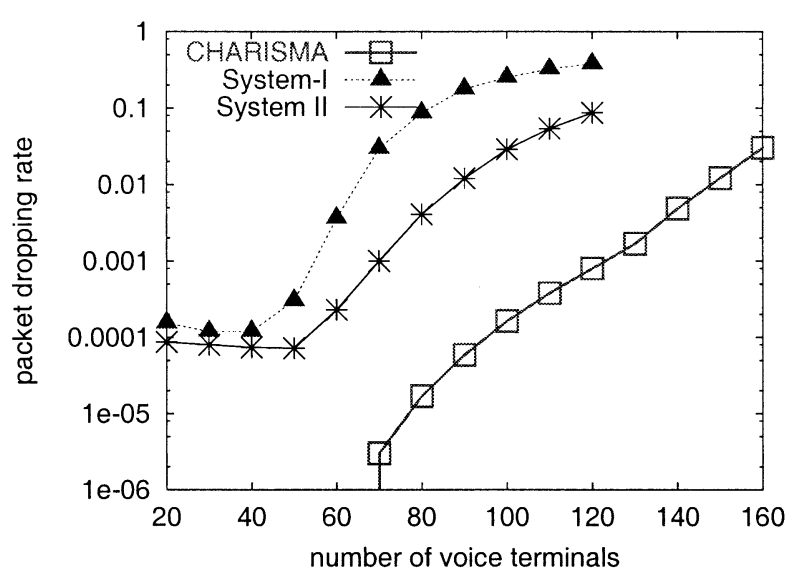

(a) $N_{d}=0$

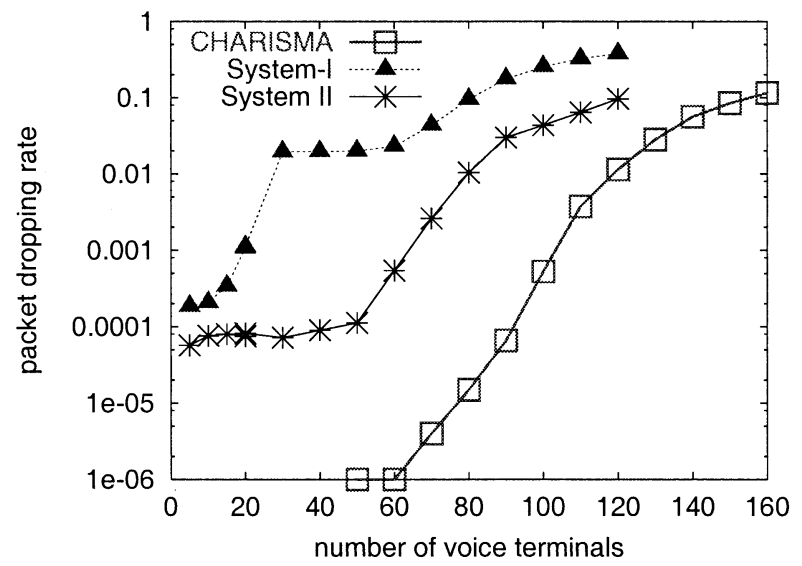

(b) $N_{d}=10$

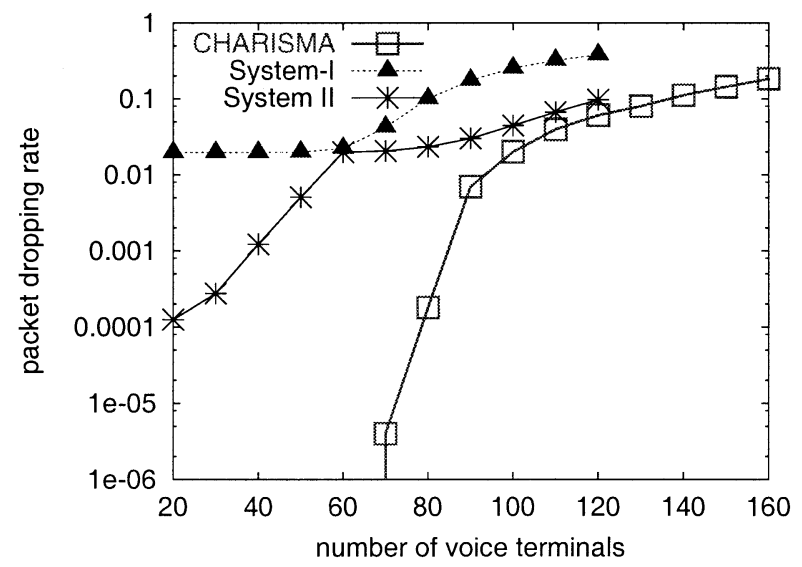

(c) $N_{d}=20$

Fig. 13. Illustration of synergistic effect-voice terminal performance comparison (without capture).

twice the offered throughput relative to System-I. This illustrates the merit of using a variable-rate physical layer. However, the potential of synergistic gain is demonstrated by the significant improvement in terms of packet-dropping probability under the same traffic load of CHARISMA relative to System-II. For example, at the $1 \%$ voice packet dropping rate threshold, the number of voice terminals that can be accommodated by CHARISMA is 2.5 times and 1.87 times greater than those

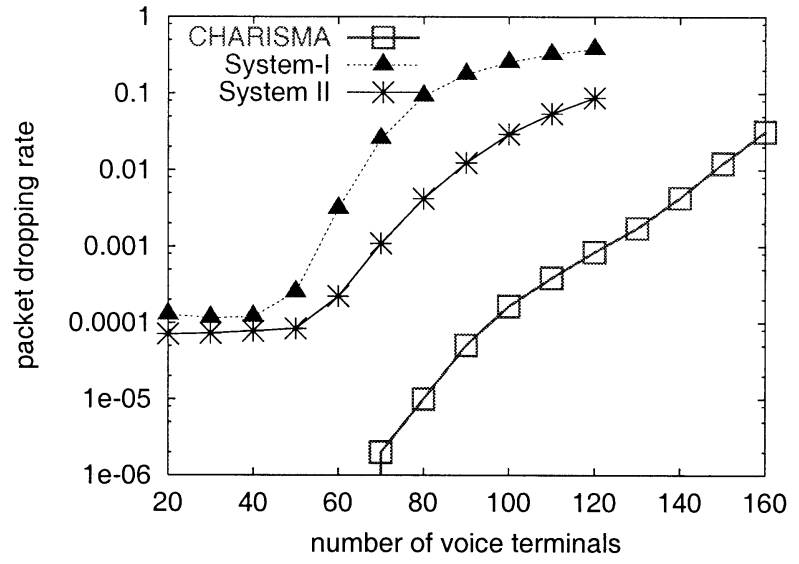

(a) $N_{d}=0$

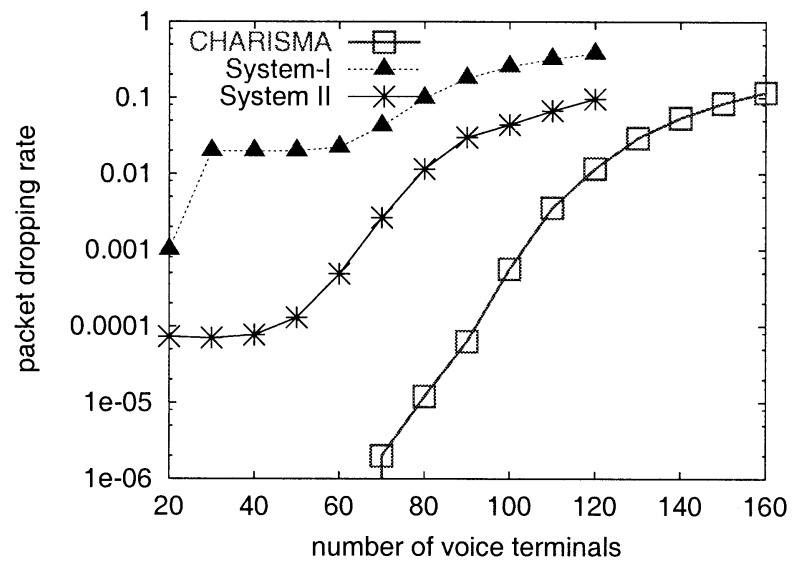

(b) $N_{d}=10$

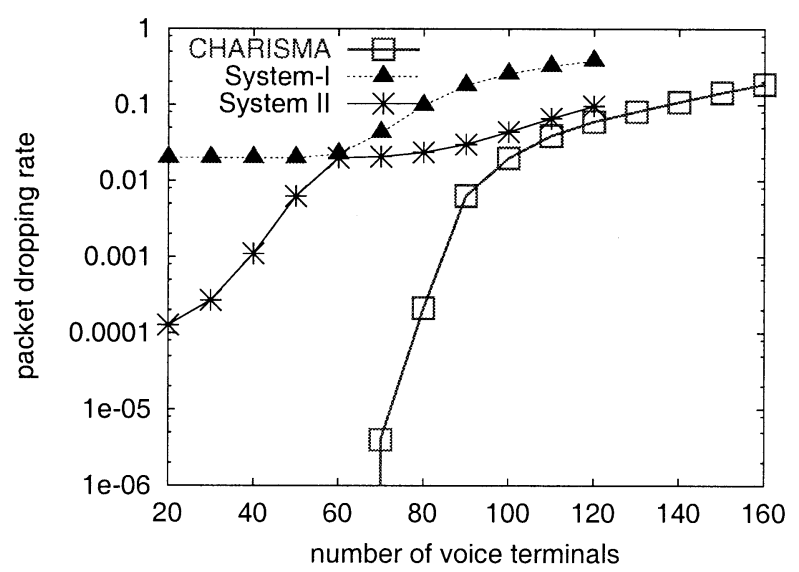

(c) $N_{d}=20$

Fig. 14. Illustration of synergistic effect-voice terminal performance comparison (with capture).

of System-I and System-II, respectively, for cases without capture and $N_{d}=0$ [see Fig. 13(a)]. For cases where there are data users, the CHARISMA protocol outperforms the two baseline systems by an even greater margin. Indeed, as shown in Fig. 13(c) (20 data users), CHARISMA can support 10 times and two times more voice terminals compared with System-I and System-II, respectively. The performance results for cases with capture show a similar trend. These results indicate that the capacity of the 


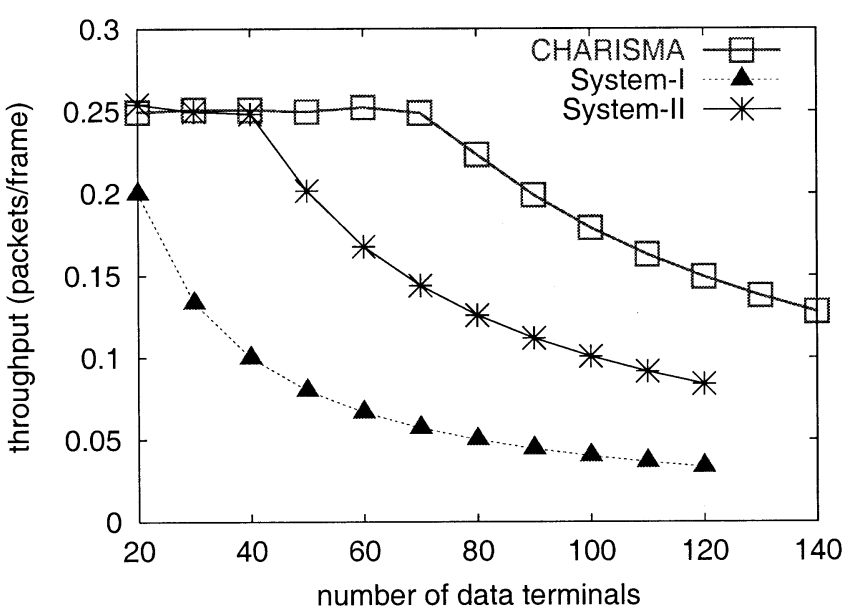

(a) $N_{v}=0$

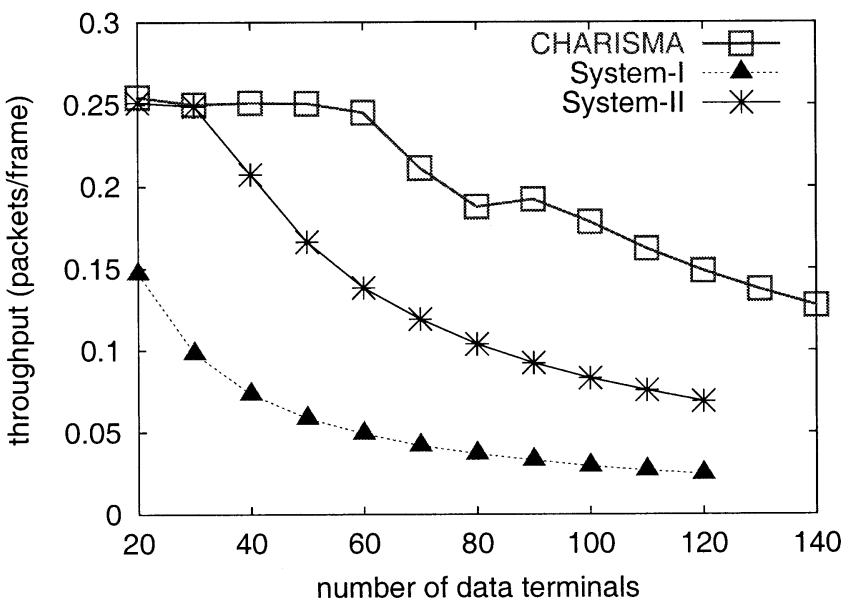

(b) $N_{v}=20$

Fig. 15. Data terminal performance-throughput against traffic load (without capture).

system is substantially increased by the synergistic interaction between the MAC and physical layers.

\section{B. Performance for Data Terminals}

Data packets are delay insensitive, and as such, they will not be discarded at the transmitter. However, they may experience transmission errors when the channel state is too bad. Lost data packets are retransmitted (through the datalink layer). This introduces additional retransmission delay. To quantify the data terminal performance, we use the average throughput and the delay as the performance measures. The average data throughput $\bar{\rho}$ is defined as the average number of data packets successfully received at the base station per frame per user. The average delay $\bar{D}_{d}$ is defined as the average time that a data packet spends waiting in the buffer until the beginning of the successful transmission. Figs. 15-18 illustrate the performance of the data terminal in terms of $\bar{\rho}$ and $\bar{D}_{d}$, respectively. When the traffic load is high, the system is in a highly congested state, so that the average per-user throughput drops and the average per-user delay increases dramatically. These adverse phenomena are detrimental to the data users' quality of service (QoS), which depends critically on the parameters pair (delay, throughput). Again, in all test cases, System-II is superior to

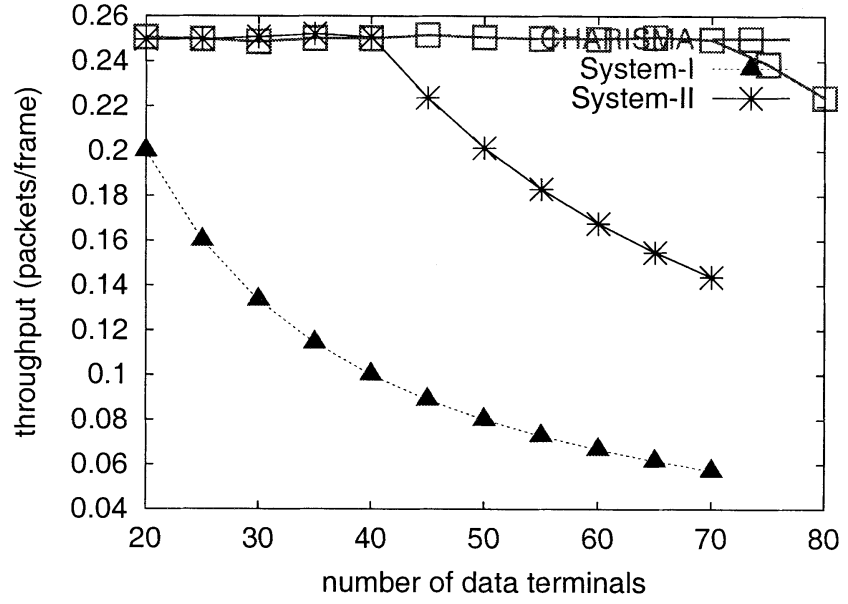

(a) $N_{v}=0$

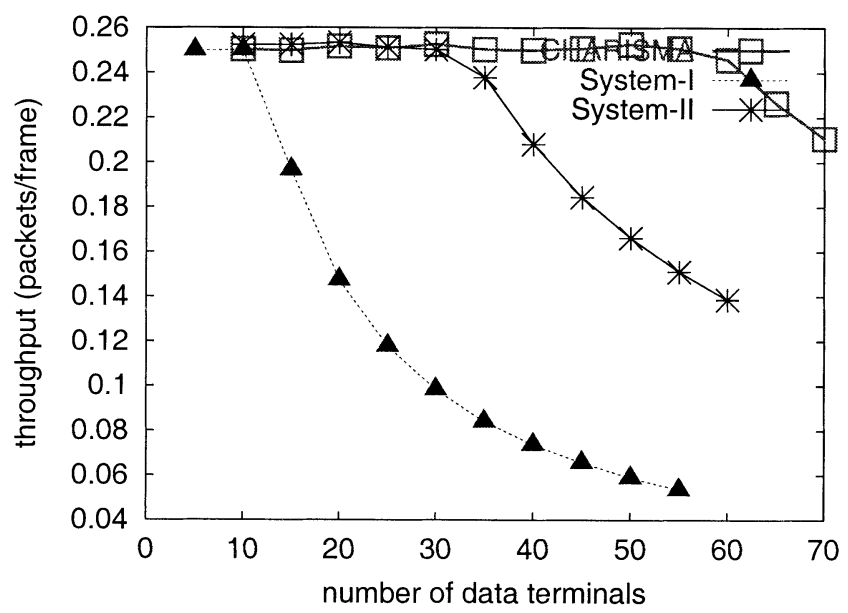

(b) $N_{v}=20$

Fig. 16. Data terminal performance-throughput against traffic load (with capture).

System-I in terms of $\bar{\rho}$ and $\overline{D_{d}}$ because the former has twice the average offered throughput from the channel-adaptive physical layer. However, the remarkable synergistic effect is again demonstrated by the performance gains of the proposed CHARISMA protocol relative to System-II. For example, at a QoS level of (1 s, 0.25), the number of data users that can be accommodated by CHARISMA is 14 times and two times greater than those of System-I and System-II, respectively, for test cases without capture and $N_{v}=0$. Furthermore, for test cases with 20 voice users, the data user capacity of CHARISMA is 60 times and 2.2 times greater than those of System-I and System-II, respectively. The results with capture concur with these observations.

\section{Interpretations}

Synergy is clearly demonstrated by the experiment results described above. The substantial performance improvements are explicable by the following arguments.

1) A voice terminal may experience a deep fading for a long time when it is affected by shadowing. In System-I or System-II, bandwidth allocation in the MAC layer is carried out regardless of the current channel condition as detected in the physical layer. Thus, information slots 


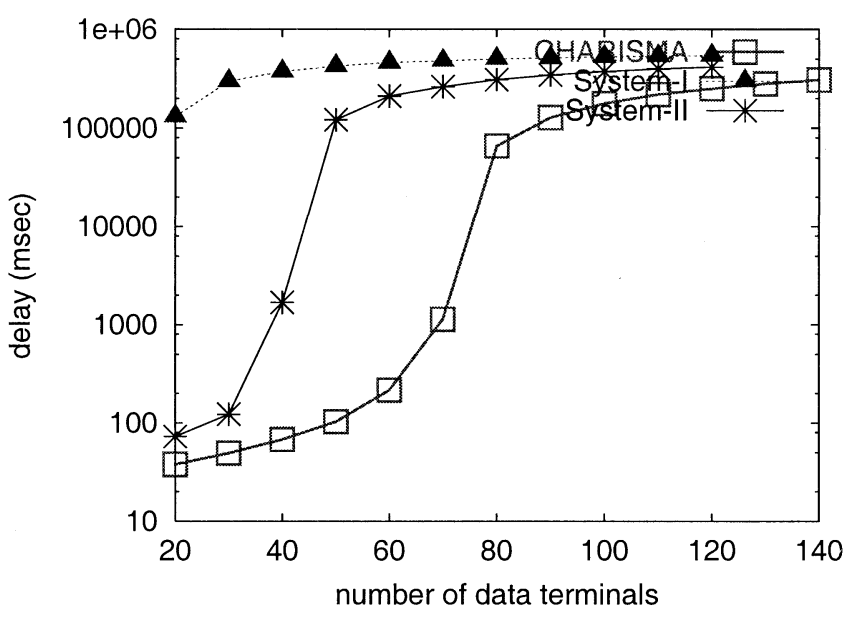

(a) $N_{v}=0$

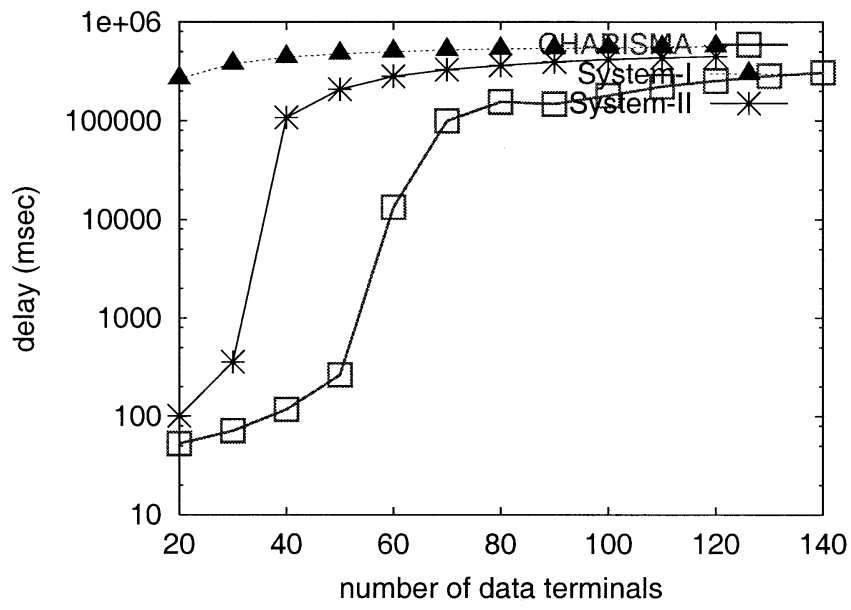

(b) $N_{v}=20$

Fig. 17. Data terminal performance-delay against traffic load (without capture).

could also be allocated to such a user, and the transmitted packets will be very likely lost due to the poor channel condition. In other words, assigned slots are simply wasted. This kind of wasteful allocation is avoided in our proposed CHARISMA protocol.

2) Selection diversity is implicitly incorporated in the CHARISMA protocol. Through the priority-based assignment process, every frame is packed with a selected group of information packets with good channel states. Thus, the effective delivered throughput per frame achieved in CHARISMA can be much higher than that in System-II. In CHARISMA, a large number of transmission requests are collected first before allocation of information slots. From the collection of requests, there is a high likelihood that a sufficient number of requests with good channel states can be selected to fully utilize the information slots in an effective manner (i.e., high throughput). For those requests with poor instantaneous channel states, their transmissions are deferred until the CSI improves or the deadlines are approaching. By contrast, in System-II, requests are served in a first-come, first-served manner due to the traditional strategy of immediately assigning slots upon successful receipt of requests. Thus, the channel states of such requests are

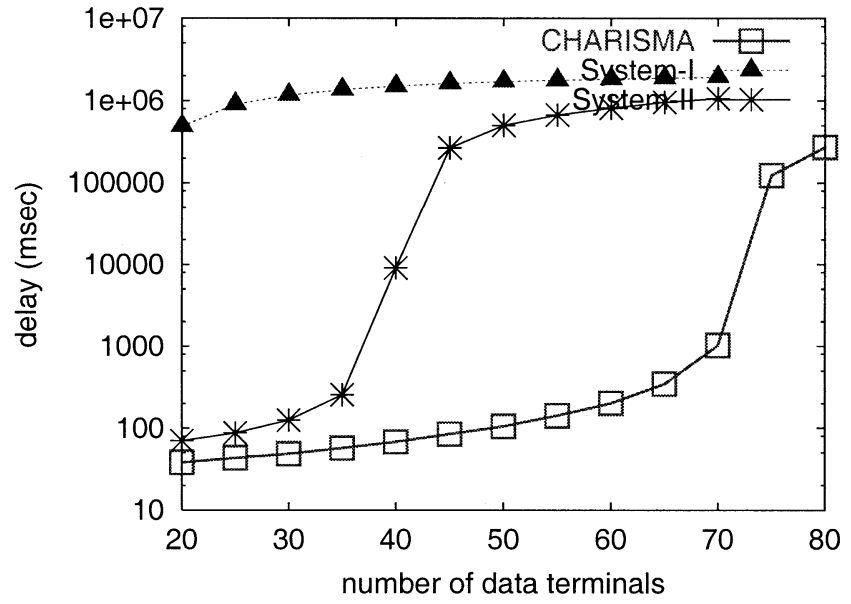

(a) $N_{v}=0$

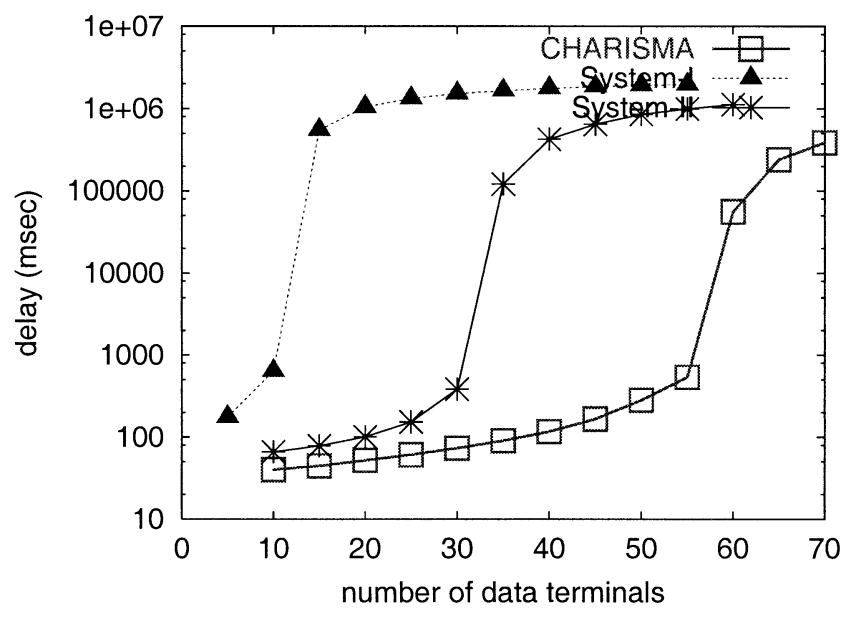

(b) $N_{v}=20$

Fig. 18. Data terminal performance—delay against traffic load (with capture).

highly diverse; and, most importantly, some requests with bad channel states (hence very low throughput) are also served, thereby causing inefficient the bandwidth utilization. For example, a voice terminal may experience a very good CSI for a long time (out of shadowing). In System-I or System-II, however, this user may fail to successfully transmit a request to the base station, probably because of excessive collisions in the request phase. In comparison, our proposed scheme gathers a large number of requests through successive frames and allocates time slots to the users that can use the system bandwidth more effectively. Thus, the likelihood of "missing" a user with good channel state is much lower, and the utilization of bandwidth is therefore higher.

3) In general, the performance improvements with capture are slightly reduced compared to the situation without capture because the capture effect helps improve System-II. Specifically, close scrutiny of the capture effect reveals that a request with a better CSI (i.e., higher received power) is more likely to be captured than a request with a worse CSI (i.e., lower received power). Thus, the capture process itself serves as a channel state selector-a request that has a higher throughput will be more likely to be chosen for slot assignment. 


\section{CONCLUSION}

While the layering and information-hiding design paradigm is useful for managing the complexity of communication protocols, to achieve high performance, synergy between protocol layers has to be exploited. In this paper, using our proposed channel adaptive MAC protocol, we illustrate that such synergistic effect can indeed deliver considerable performance improvement. The proposed protocol, called CHARISMA, employs a variable-throughput adaptive channel encoder and modulator in the physical layer. The MAC layer is a dynamic TDMA-based algorithm in which a transmission frame is divided into a request phase and an information phase. Synergy is achieved by the interaction between the physical layer and the MAC layer. Specifically, the physical layer offers a variable throughput to the MAC layer depending on the current channel condition. Accordingly, the MAC layer allocates bandwidth by taking into account of the current throughput level of the physical layer. Our extensive simulation studies indicate that significant performance gains for voice terminals and data terminals are achieved in the CHARISMA protocol. Given these encouraging results, we are currently investigating the extension of the synergy concept to higher protocol layers for wireless communication.

\section{ACKNOWLEDGMENT}

The authors would like to thank the anonymous reviewers for their comments on this paper.

\section{REFERENCES}

[1] M. B. Abbott and L. L. Peterson, "Increasing network throughput by integrating protocol layers," IEEE/ACM Trans. Networking, vol. 1, pp. 600-610, Oct. 1993.

[2] I. F. Akyildiz, J. McNair, L. C. Martorell, R. Puigjaner, and Y. Yesha, "Medium access control protocols for multimedia traffic in wireless networks," IEEE Network, vol. 13, no. 4, pp. 39-47, July/Aug. 1999.

[3] S. M. Alamouti and S. Kallel, "Adaptive trellis-coded multiple-phaseshift keying for Rayleigh fading channels," IEEE Trans. Commun., vol. 42, pp. 2305-2314, June 1994.

[4] J. C. Ambak and W. van Blitterswijk, "Capacity of slotted ALOHA in Rayleigh fading channels," IEEE J. Select. Areas Commun., vol. SAC-5, pp. 261-268, Feb. 1987.

[5] N. Amitay and L. J. Greenstein, "Resource Auction Multiple Access (RAMA) in the cellular environment," IEEE Trans. Veh. Technol., vol. 43, pp. 1101-1111, Nov. 1994.

[6] P. Bhagwat, P. Bhattacharya, A. Krishna, and S. K. Tripathi, "Using channel state dependent packet scheduling to improve TCP throughput over wireless LAN's," ACM/Baltzer Wireless Networks, vol. 3, no. 1, pp. 91-102, 1997.

[7] A. Chockalingam, M. Zorzi, and V. Tralli, "Wireless TCP performance with link layer FEC/ARQ," in Proc. ICC'99, June 1999, pp. 1212-1216.

[8] G. Falk, J. S. Groff, W. C. Milliken, M. Nodine, S. Blumenthal, and W. Edmond, "Integration of voice and data in the wideband packet satellite network," IEEE J. Select. Areas Commun., vol. SAC-1, pp. 1076-1083, Dec. 1983.

[9] D. J. Goodman, "Trends in cellular and cordless communications," IEEE Commun. Mag., vol. 29, pp. 31-40, June 1991.

[10] D. J. Goodman, R. A. Valenzuela, K. T. Gayliard, and B. Ramamurthi, "Packet reservation multiple access for local wireless communications," IEEE Trans. Commun., vol. 37, pp. 885-890, Aug. 1989.

[11] J. Gruber and L. Strawczynski, "Subjective effects of variable delay and speech clipping in dynamically managed voice systems," IEEE Trans. Commun., vol. COM-33, pp. 801-808, Aug. 1985.

[12] M. Inoue, G. Wu, and Y. Hase, "Channel state dependent resource scheduling for wireless message transport," in Proc. VTC'98, 1998, pp. 1264-1268.
[13] D. G. Jeong, C.-H. Choi, and W. S. Jeon, "Design and performance evaluation of a new medium access control protocol for local wireless data communications," IEEE/ACM Trans. Networking, vol. 3, pp. 742-752, Dec. 1995.

[14] M. Kawagishi, S. Sampei, and N. Morinaga, "A novel reservation TDMA based multiple access scheme using adaptive modulation for multimedia wireless communication systems," in Proc. VTC'98, 1998, pp. 112-116.

[15] Y. S. Liu and S. W. Wu, "Adaptive coding scheme for wireless LAN's with CSMA/CA MAC protocol," Electron. Lett., vol. 34, no. 15, pp. $1450-1452,1998$.

[16] S. Y. Mui, "A comparison of fixed and variable-rate signalling for meteor burst communications," IEEE Trans. Commun., pp. 211-215, Feb.-Apr. 1994.

[17] D. Cygan and E. Lutz, "A concatenated two-stage adaptive (CTSA) error control scheme for data transmission in time-varying channels," IEEE Trans. Commun., vol. 43, pp. 795-803, Feb.-Apr. 1995.

[18] "Guidelines for Evaluation of Radio Transmission Technologies for IMT-2000,”, ITU-R M.1225, 1997.

[19] V. K. N. Lau, "Performance analysis of variable rate: symbol-by-symbol adaptive bit interleaved coded modulation for Rayleigh fading channels," in IEEE Trans. Veh. Technol., vol. 51, May 2002, pp. 537-550.

[20] V. K. N. Lau and Y.-K. Kwok, "Synergy between adaptive channel coding and media access control for wireless ATM," in Proc. VTC'99 Fall, Sept. 1999.

[21] V. K. N. Lau and S. V. Maric, "Variable rate adaptive modulation for DS-CDMA," IEEE Trans. Commun., vol. 47, pp. 577-589, Apr. 1999.

[22] X. Qiu and V. O. K. Li, "Dynamic reservation multiple (DRMA): A new multiple access scheme for personal communication system (PCS)," ACM/Baltzer Wireless Networks, vol. 2, pp. 117-128, 1996.

[23] A. Urie, M. Streeton, and C. Mourot, "An advanced TDMA mobile access system for UMTS," in Proc. IEEE PIMRC'94, 1994.

[24] M. D. Yocoub, Foundations of Mobile Radio Engineering, 2nd ed. New York: McGraw-Hill, 1986.

[25] K. J. Zdunek, D. R. Ucci, and J. L. LoCicero, "Packet radio performance of inhibit sense multiple access with capture," IEEE Trans. Commun., vol. 45, pp. 164-167, Feb. 1997.

[26] M. Zorzi and R. R. Rao, "The role of error correlations in the design of protocols for packet switched services," in Proc. 35th Annu. Allerton Conf. Commuincations, Control and Computing, Sept. 1997, pp. 749-758.

Vincent K. N. Lau (M'98-SM'01) received the B.Eng. degree (with first class honors) in electrical engineering from the University of Hong Kong in 1992 and the Ph.D. degree from the University of Cambridge, Cambridge, U.K., in 1997.

He was with HK Telecom for three years as a System Engineer responsible for transmission systems design.He joined the Lucent Technologies-Bell Labs Member of Technical Staff, where he was engaged in the algorithm design, standardization, and prototype development of CDMA2000 systems. He joined the University of Hong Kong in 1999 as an Assistant Professor and became Codirector of the Information Engineering Program as well as Codirector of the 3G Technology center. In 2001, he returned to the Wireless Advanced Technology Lab of Lucent Technologies. His research interests include digital transceiver design, adaptive modulation and channel coding, CDMA power control, soft handoff and CREST factor control algorithms, jointly adaptive multiple-access protocols, and short-range wireless ad hoc networking. He is currently working on BLAST-MIMO systems, iterative decoding, and UMTS call processing protocol stack design.

Dr. Lau received the Sir Edward Youde Memorial Fellowship and the Croucher Foundation in 1995.

Yu-Kwong Kwok (S'94-M'95) received the B.Sc. degree in computer engineering from the University of Hong Kong in 1991 and the M.Phil. and Ph.D. degrees in computer science from the Hong Kong University of Science and Technology in 1994 and 1997, respectively.

$\mathrm{He}$ is an Associate Professor in the Department of Electrical and Electronic Engineering, University of Hong Kong. He was a Visiting Scholar for one year in the Parallel Processing Laboratory, School of Electrical and Computer Engineering, Purdue University, West Lafayette, IN. His research interests include adaptive wireless communication protocols, mobile computing based on shortrange wireless technologies, software support for parallel and distributed computing, heterogeneous cluster computing, and distributed multimedia systems.

Prof. Kwok is a member of the ACM, the IEEE Computer Society, and the IEEE Communications Society. 